\title{
Pectus excavatum requiring temporary pacemaker implantation before Nuss procedure: a case report
}

\author{
Gebang Wang ${ }^{1}$, Zhanwu Yu ${ }^{1}$, Chenlei Zhang ${ }^{1}$, Hongyun Zang ${ }^{2}$, Lorenzo Monti ${ }^{3}$, Jin Yong Jeong ${ }^{4}$, \\ Ralph A. Schmid ${ }^{5}$, Hans K. Pilegaard ${ }^{6}$, Hongxu Liu ${ }^{1}$
}

${ }^{1}$ Department of Thoracic Surgery, Cancer Hospital of China Medical University, Liaoning Cancer Hospital \& Institute, Shenyang, China; ${ }^{2}$ Department of Cardiology, Air Force Hospital of North Military Command PLA, Shenyang, China; ${ }^{3}$ Radiology Department, Humanitas Research Hospital, Milan, Italy; ${ }^{4}$ Department of Thoracic and Cardiovascular Surgery, College of Medicine, The Catholic University of Korea, Seoul, Korea; ${ }^{5}$ Division of General Thoracic Surgery, University Hospital Bern, Freiburgstrasse, CH-3010 Bern, Switzerland; ${ }^{6}$ Department of Cardiothoracic and Vascular Surgery, Aarhus University Hospital, Aarhus and Institute of Clinical Medicine, Aarhus University, Aarhus, Denmark

Correspondence to: Hongxu Liu. Department of Thoracic Surgery, Cancer Hospital of China Medical University, Liaoning Cancer Hospital \& Institute, No. 44 Xiaoheyan Road, Shenyang 110042, China. Email: hongxuliu@qq.com.

\begin{abstract}
Pectus excavatum is the most common chest wall deformity, and some patients also have it combined with cardiac arrhythmias. It is a rare occurrence for there to be a severe conduction block that requires a temporary pacemaker implantation before the surgical correction. Here we reported a case of pectus excavatum with a second-degree atrial-ventricular (AV) block (Mobitz II) who had temporary pacemaker implantation before the Nuss procedure. The young patient had a chest wall deformity for 6 years and it got worse with age. The Haller index was 4.21, and we evaluated that he should receive the Nuss procedure. An AV block was found during the preoperative electrocardiogram examination; furthermore, Holter monitor proved that he had first-degree AV block and a second-degree AV block (Mobitz II). After consultation with the anesthesiologist and cardiologist, we suggested that a temporary pacemaker placement should be performed under local anesthesia before the minimally invasive operation and removed as soon as the patient revived from general anesthesia. A postoperative Holter monitor was implemented, and the conduction defect disappeared shortly after the operation. However, the Holter monitor showed that the conduction defect was still existed during the follow-up period, which indicated that severe conduction defects should be originated from the conduction system itself, rather than the compression to the heart. The temporary pacemaker was essential to ensure the conducting of the operation went smoothly.
\end{abstract}

Keywords: Pectus excavatum; temporary pacemaker implantation; second-degree atrial-ventricular block; Nuss procedure

Submitted Feb 04, 2020. Accepted for publication Aug 21, 2020.

doi: $10.21037 /$ jtd-20-2312

View this article at: http://dx.doi.org/10.21037/jtd-20-2312

Pectus excavatum (PE) is the most common chest wall deformity (1). Patients may complain of exercise intolerance, dyspnea, chest pain, or palpitations (2), severe symptoms like syncope are rare (3). Preoperative examinations could reveal distinct types of cardiac arrhythmia. However, there were no reports of combining with second-degree atrial-ventricular (AV) block (Mobitz II). Furthermore, pectus excavatum requiring temporary pacemaker implantation for the sake of a combination of the AV block before the operation was infrequent.

We here presented a case of pectus excavatum combined with a second-degree AV block (Mobitz II), which also required a temporary pacemaker implantation before the Nuss procedure.

We present the following case in accordance with the CARE reporting checklist (available at http://dx.doi. org/10.21037/jtd-20-2312). 


\section{Case presentation}

A 17-year-old adolescent boy was admitted to our department with a severe asymmetric pectus excavatum, Haller index, 4.21 (Figure 1). His parents found the chest wall deformity six years ago, and the deformity got worse and symptoms developed with his growth. After routine physical examinations, we considered that he should receive the Nuss procedure.

The results of the preoperative examinations were within normal limits except for electrocardiogram, which showed first-degree AV block. Another Holter monitor was performed, showed that first-degree AV block, seconddegree AV block (Mobitz II, Figure 2). The patient had no cardiac-related symptoms. A consultation with the anesthesiologist and cardiologist was promoted, and finally we decided that a temporary pacemaker implantation should

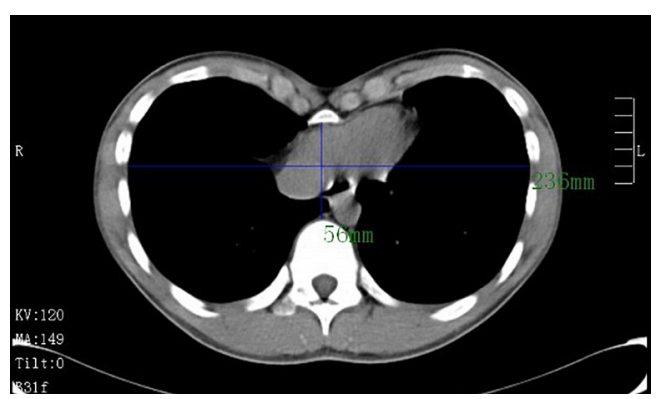

Figure 1 The chest CT showed the heart was compressed by the sternum with the Haller index 4.21 . be performed under local anesthesia before the minimally invasive (Nuss) operation and removed when the patient recovered from general anesthesia.

The operation went ahead as planned. The temporary pacemaker was placed from his right femoral vein to the cardiac apex under local anesthesia, and the Nuss procedure under video-assisted thoracoscope (VATS) was performed after. An incision about $4 \mathrm{~cm}$ between the anterior and midaxillary line in each side of chest wall was made at the most severe compression level based on the length of the bar. One bar and a stabilizer on the right chest wall were implanted from the highest points of the "funnel" deformity, immobilized on the ribs exactly with satisfactory deformity correction (Figure 3). During the operation, the atrial pacemaker was activated due to transient heart rate decline. The patient recovered well with no postoperative perioperative complications. The Holter monitor on postoperative day 5 showed that there was no first- or second-degree AV block, and he was discharged from our department on the 6th postoperative day. He was followedup after 24 months, the correction of the deformity was perfect, but it was a pity that the second-degree AV block (Mobitz II) was still existed without any cardiac-related symptoms, which indicated that the arrhythmia was cardiac originated.

All procedures performed in studies involving human participants were in accordance with the ethical standards of the institutional and/or national research committee(s) and with the Helsinki Declaration (as revised in 2013). Written
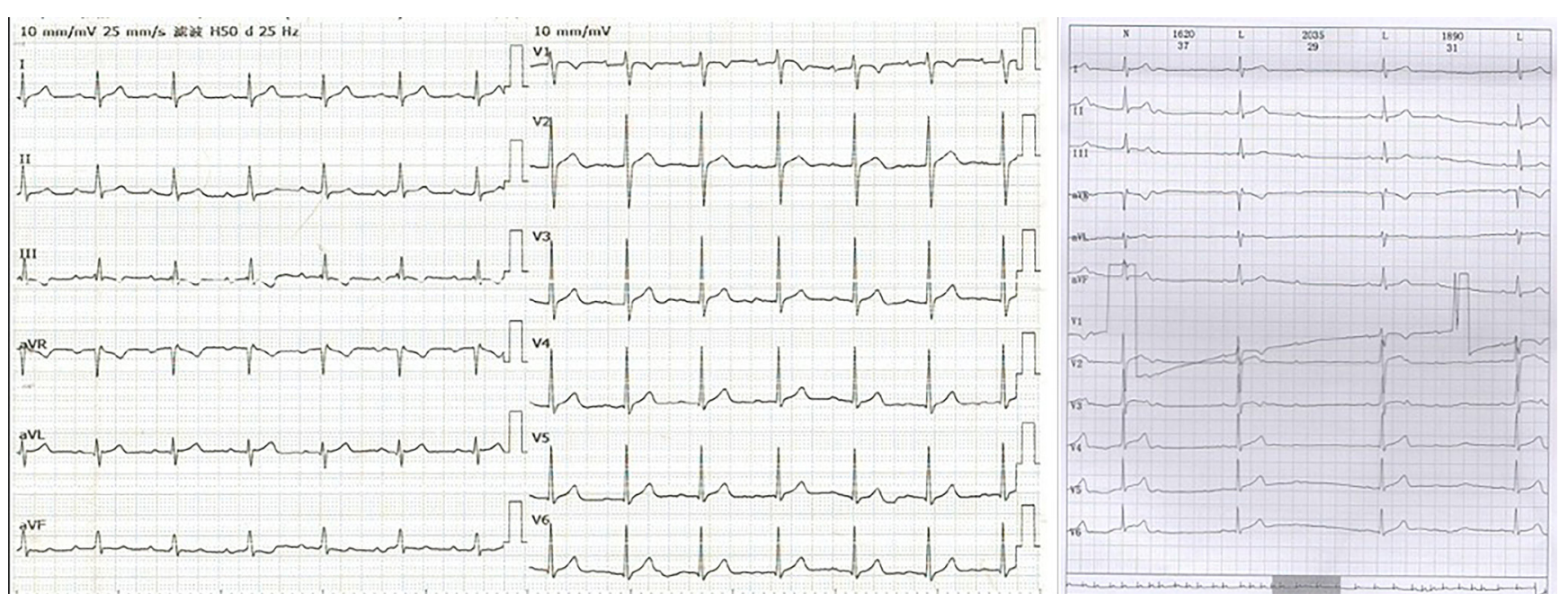

Figure 2 The electrocardiogram (ECG) and 24-hour Holter showed that the patient had first-degree AV block, second-degree atrialventricular block (AV block) (Mobitz II). 

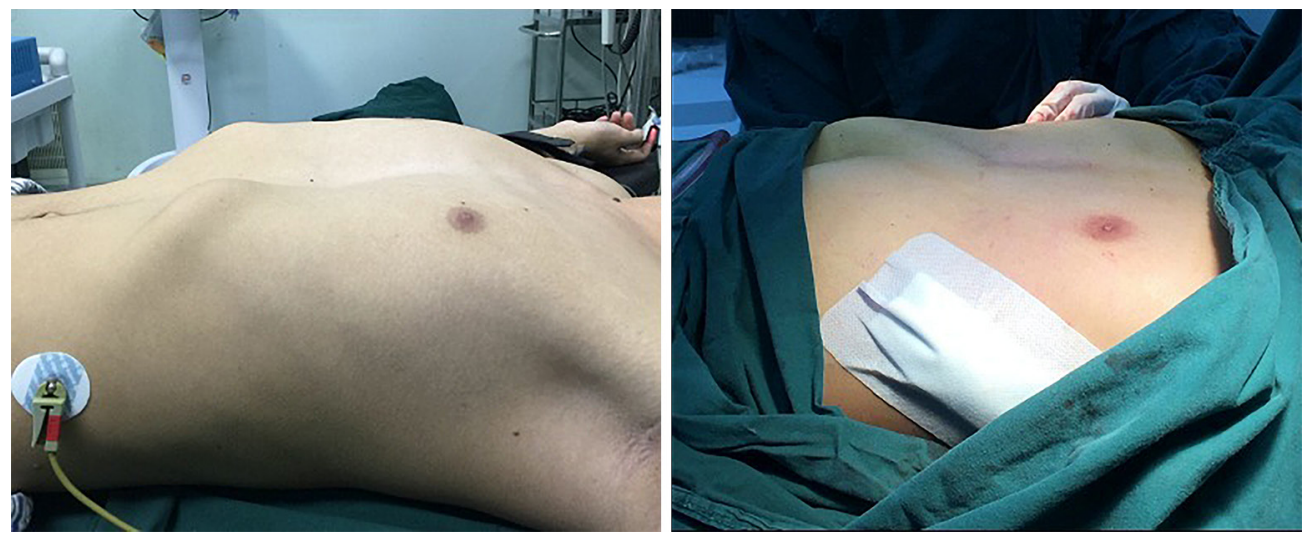

Figure 3 The surgical correction of the chest wall deformity was successful through Nuss procedure.

informed consent was obtained from the patient.

\section{iMDT discussion}

\section{Discussion among physicians from local hospitals}

As the most common chest wall abnormality, pectus excavatum accounts for more than $90 \%$ of all congenital chest wall abnormalities (1). Most patients are healthy and the most common symptoms include exercise intolerance, dyspnea, chest pain, or palpitations (2). The only physiologic explanation for these complaints is the compression of chest wall malformation to the lung and the heart, since the pulmonary parenchyma and airways of the lung are not generally diseased (4).

Some patients could be found distinct types of cardiac arrhythmia through electrocardiogram examination. Literature has reported that cardiac arrhythmias including first-degree heart block, right bundle branch block, WolffParkinson-White syndrome, ventricular tachycardia, Brugada phenocopies, atrial fibrillation, incomplete left bundle branch block, are life-threatening (5-10). Also, electrocardiography of patients with $\mathrm{PE}$ was differed from patients to patients (11). Most arrhythmias relieved after the correction of the deformity without recurrence during follow-up. Our operation team has treated more than 150 pectus excavatum cases with the Nuss procedure, and it was the first time that we encountered severe cardiac arrhythmia.

\section{Department of Anesthesiology}

The patient was asymptomatic with severe cardiac arrhythmia. A temporary pacemaker could be a protective measure used for noncardiac surgery under general anesthesia during the perioperative period for patients with severe conduction defects. According to ACC/AHA Guidelines on Perioperative Cardiovascular Evaluation and Care for Noncardiac Surgery (12), temporary pacemaker implantation during the perioperative period for protective or preventative pace-making could improve the security of anesthesia and operation.

\section{Department of Cardiology}

For this patient, he suffered from severe cardiac arrhythmia. It is an indication that second-degree AV block (Mobitz II) patient should receive pacemaker implantation, even for permanent, except for asymptomatic patients. It is proper to have the pacemaker to protect the patient from cardiac arrest during operation. The temporary pacemaker stopped when using the electrocoagulation for mobilization or hemostasis. His abnormal dysrhythmia persisted after deformity correction, which represents that the dysrhythmia derived from the cardiac conduction system. For patients who had second-degree AV block with no clinical symptoms, it is recommended that the patient should consult cardiologists for a further and detailed examination to determine whether he should undergo temporary or permanent cardiac pacemaker implantation.

\section{Several issues regarding the diagnosis and treatment of this patient were further discussed as follows}

For patients of pectus excavatum with cardiac arrhythmias, how to distinguish the originations of the heart diseases?

\section{Expert opinion 1: Dr. Hans K. Pilegaard}

Most patients with pectus excavatum are treated when 
they are young (less than 20 years) so the risk of a cardiac arrhythmia caused by the pectus is minimal and exceptional and the evidence that a pectus causes arrhythmias or blocks the cardiac conduction system is weak. This means that finding these symptoms might be caused by a heart disease.

In this case, a 17-year-old with a moderate pectus excavatum without any cardiac symptoms, we do not agree in the conclusion concerning the block. We find that the ECG is normal. The discretely prolonged PR-interval is normal in healthy young individuals, and may represent increased vagal tone. We find that the rhythm strip more likely shows Wenckeback block (AV block type I) and not type II as proposed. Before the first QRS complex there is a significant prolonged $\mathrm{PR}$-interval followed by a $\mathrm{P}$-wave that is not transmitted causing a drop out of one QRS-complex. After that every second $\mathrm{P}$-wave is blocked. This finding is not sufficient for making a diagnosis of type II grade II AV-block. That at minimum requires that two consecutive waves are blocked. So the findings are not necessarily pathological and do not require pacing neither temporary or permanently. The findings are common in healthy (often well-trained) young people.

\section{Expert opinion 2: Dr. Fin Yong feong}

There are cases of patients of pectus excavatum accompanying cardiac arrhythmia. The cardiac arrhythmia, caused by pectus excavatum, has been known it would be attributable to the dilatation of right atrium induced by the increase of right ventricular pressure resulted from right ventricle depressed by sunken sternum. There are representative symptoms of arrhythmia such as atrial flutter and atrial fibrillation. In cases of finding such symptoms of arrhythmia from patients of pectus excavatum in the age of predilection (less than 55 years of age), the arrhythmia could possibly be attributed to the presence of pectus excavatum more frequently.

There are also cases of pectus excavatum which were reported with the occurrence of WPW, first-degree AV block, and RBBB, or accompanying ventricular tachycardia. However, the attribution of all arrhythmia to pectus excavatum seems unreasonable.

In this case, the second-degree AV block (Mobitz type 2), found before the operation of pectus excavatum, remained postoperatively after completion of the operation. On the contrary, the cases of the relief from supraventricular arrhythmia or ventricular tachycardia, that accompanied the pectus excavatum, are occasionally reported with the completion of the operation of pectus excavatum. Therefore, in the cases of finding favorable turns of symptoms of arrhythmia upon completion of operation of pectus excavatum, the causes of arrhythmia could be discriminated as findings suggesting pectus excavatum rather than cardiac disorders.

\section{Expert opinion 3: Dr. Lorenzo Monti}

Conduction disorders in childhood are rare and their cause should always be sought by excluding a family history, rheumatologic diseases (SLE or scleroderma), infiltrative processes (amyloidosis, sarcoidosis) or neurodegenerative processes.

Although some level of association between pectus excavatum and cardiac arrhythmias has been reported in literature $(3,9,13)$, there is no clear cause-effect relationship between chest wall abnormalities and advanced AV-block, neither evidence-based guidelines to help the interpretation of cardiac arrhythmias in $\mathrm{PE}$ patients.

In PE patients with arrhythmias, an echocardiographic study is warranted. A tricuspid regurgitation with right atrial enlargement eventually leading to atrial fibrillation (10) is likely to be solved with a Nuss operation.

In $\mathrm{PE}$ patients with frequent premature ventricular contractions and negative precordial $\mathrm{T}$ waves (possibly related to the clockwise rotation of the heart) the differential diagnosis with Arrhythmogenic Right Ventricular Cardiomyopathy may be tricky and a Cardiac Magnetic Resonance will help.

\section{Expert opinion 4: Dr. Ralph A. Schmid}

It depends of the type of the arrhythmia. If you find for example symptomatic ventricular tachycardia, I would be careful and extend monitoring during the perioperative period. Extended cardiac evaluation with mapping would be feasible to exclude other reasons than PE. And echocardiography or cardiac MRI to exclude valvular disorders or other anatomical abnormalities. It also depends on the frequency of the episodes of the arrhythmia. It is a different story, if they occur several times a day or only once in a few weeks.

\section{What kinds of cardiac diseases for the patients combined with should have the pacemaker implantation, and what's the time of removal the pacemaker, and what are the indications for permanent pacemaker implantation?}

\section{Expert opinion 1: Dr. Hans K. Pilegaard}

Patients should receive pacemaker treatment according to the normal guidelines for pacemaker implantation.

Expert opinion 2: Dr. Fin Yong Feong

The chances of pacemaker implantation due to AV block 
or sick sinus syndrome associated with pectus excavatum are low. However, implantation of temporary pacemaker seems plausible because the anesthesia may aggravate the symptoms of arrhythmia. The duration of implantation would be dependent upon causative factors, however, we presume it would extend at best a week since the temporary pacemaker is difficult to fix thereby discomforts patients seriously. In general, the permanent pacemaker would be efficacious to patients of the symptoms without reversible cause. Thus, it would be applicable to cases of type 2 second-degree AV block, high grade AV block, and complete AV block occurred without reversible cause.

\section{Expert opinion 3: Dr. Lorenzo Monti}

In a recently published 20-years national registry, Mobitz II AV block account for $19 \%$ of permanent pacing in young patients (14), but pectus excavatum is not listed as a possible cause of permanent pacing.

In asymptomatic patients with advanced AV block without specific risk conditions, permanent pacing is not indicated. In this case, however, it was reasonable to ensure a temporary pacing during the surgical time.

There is no current evidence that arrhythmias in patients with pectus excavatum may benefit from a specific management. Therefore, both implantation and follow-up timing need to adhere to the current guidelines (15).

\section{Expert opinion 4: Dr. Ralph A. Schmid}

In most cases, I guess, not a pacemaker is necessary, but a defibrillator, as ventricular arrhythmia is the most common arrhythmia associated with PE. In most cases a permanent pacemaker is not necessary. If there would be no arrhythmia over a time span of 2 to 3 weeks, I would remove the device (defibrillator or pacemaker).

A temporary device (pacemaker or defibrillator) would have to be installed preoperatively to allow therapy or support if the arrhythmia occurs intraoperatively. Close collaboration with the anesthesiologist is mandatory.

\section{What are the advices for the patient during follow-up period?}

\section{Expert opinion 1: Dr. Hans K. Pilegaard}

This patient who is healthy without any cardiac symptoms do not need any follow-up after the correction of the pectus excavatum but should of course be referred to a cardiologist if he later gets symptoms from the heart.

\section{Expert opinion 2: Dr. Fin Yong Jeong}

The second-degree AV block (Mobitz type 2) frequently turns into complete AV block thereby it would be necessary to take follow-up evaluation in the department of cardiology. In cases of the occurrence of symptoms such as chest pain or syncope, the patients are advised to visit hospitals to get pertinent treatments including 'reversible cause treatment' and 'permanent pacemaker implantation'.

\section{Expert opinion 3: Dr. Lorenzo Monti}

A strict cardiologic follow-up including ECG monitoringpossibly with implantable or external loop recordersshould be prescribed: a permanent pacing will be necessary in case of bradycardia-related symptoms.

\section{Expert opinion 4: Dr. Ralph A. Schmid}

One feasible possibility is long term ECG (e.g., 1 week) in the follow up period to confirm, that the arrhythmia has resolved after the correction of PE. In the immediate postoperative period, I would extent the monitoring in the intermediate care, in order to have rapid treatment, if the arrhythmia recurs.

\section{Conclusions}

As the most common type of chest wall deformities, pectus excavatum might combine with several different types of cardiac arrhythmias. Some could be relieved by the correction of the deformity, while some are originated from the heart itself. The preoperative examinations concerning the pulmonary and cardiac functions are essential for PE patients, and if any abnormal findings observed through the preoperative examinations, consultations with the related departments are recommended, and any necessary protective methods such as temporary pacemaker for patients with severe cardiac arrhythmia should be employed based on the guidelines during the correction. Patients should be referred to the specialists to follow-up in order to evaluate the condition of cardiac arrhythmias, and patients with cardiac related symptoms should be followed-up strictly and treated properly.

\section{Acknowledgments}

Funding: The work was supported by 2017 Research grant to introduced talents of Liaoning Cancer Hospital \& Institute, 2019 Grant to the Construction of Provincial Key Specialty, 2019 Guiding Program of Liaoning Natural Science Foundation.

\section{Footnote}

Reporting Checklist: The authors have completed the CARE reporting checklist. Available at http://dx.doi.org/10.21037/ jtd-20-2312 
Conflicts of Interest: All authors have completed the ICMJE uniform disclosure form (available at http://dx.doi. org/10.21037/jtd-20-2312). The authors have no conflicts of interest to declare.

Ethical Statement: The authors are accountable for all aspects of the work in ensuring that questions related to the accuracy or integrity of any part of the work are appropriately investigated and resolved. This study was approved by the Ethic Committee of Liaoning Cancer Hospital and Institute. All procedures performed in studies involving human participants were in accordance with the ethical standards of the institutional and/or national research committee(s) and with the Helsinki Declaration (as revised in 2013). Written informed consent was obtained from the patient.

Open Access Statement: This is an Open Access article distributed in accordance with the Creative Commons Attribution-NonCommercial-NoDerivs 4.0 International License (CC BY-NC-ND 4.0), which permits the noncommercial replication and distribution of the article with the strict proviso that no changes or edits are made and the original work is properly cited (including links to both the formal publication through the relevant DOI and the license). See: https://creativecommons.org/licenses/by-nc-nd/4.0/.

\section{References}

1. Shi R, Xie L, Chen G, et al. Surgical management of pectus excavatum in China: results of a survey amongst members of the Chinese Association of Thoracic Surgeons. Ann Transl Med 2019;7:202.

2. Rha EY, Kim JH, Yoo G, et al. Changes in thoracic cavity dimensions of pectus excavatum patients following Nuss procedure. J Thorac Dis 2018;10:4255-61.

3. White JA, Fine NM, Shargall Y. Pectus excavatum with compression of the inferior vena cava: a rare cause of recurrent syncope. Circulation 2009;120:1722-4.

4. Kelly RE, Goretsky MJ, Obermeyer R, et al. Twentyone years of experience with minimally invasive repair of pectus excavatum by the Nuss procedure in 1215 patients. Ann Surg 2010;252:1072-81.

5. Chan Wah Hak YS, Lim YP, Liew R, et al. Pectus excavatum: uncommon electrical abnormalities caused by extrinsic right ventricular compression. J Cardiovasc Electrophysiol 2014;25:324-7.

6. Pimenta J, Vieira A, Henriques-Coelho T. Ventricular arrhythmia solved by surgical correction of pectus excavatum. Interact Cardiovasc Thorac Surg 2018;26:706-8.

7. Awad SF, Barbosa-Barros R, Belem Lde S, et al. Brugada phenocopy in a patient with pectus excavatum: systematic review of the ECG manifestations associated with pectus excavatum. Ann Noninvasive Electrocardiol 2013;18:415-20.

8. Tran NT, Larry Klein J, Paul Mounsey J, et al. Lone atrial fibrillation is associated with pectus excavatum. Heart Rhythm 2013;10:1263-9.

9. Canpolat U, Yalçın U, Sahiner L, et al. Case images: atrial fibrillation due to right atrial compression in a patient with pectus excavatum. Turk Kardiyol Dern Ars 2012;40:392.

10. Li T, Bishop-Rimmer E, Shieh M, et al. Pectus excavatum: a cause of serious cardiac dysfunction and dysrhythmia. Am J Emerg Med 2015;33:1333.e1-2.

11. Silbiger JJ, Parikh A. Pectus excavatum: echocardiographic, pathophysiologic, and surgical insights. Echocardiography 2016;33:1239-44.

12. Fleisher LA, Fleischmann KE, Auerbach AD, et al. 2014 ACC/AHA guideline on perioperative cardiovascular evaluation and management of patients undergoing noncardiac surgery: executive summary: a report of the American College of Cardiology/American Heart Association Task Force on Practice Guidelines. Circulation 2014;130:2215-45.

13. Behr CA, Denning NL, Kallis MP, et al. The incidence of Marfan syndrome and cardiac anomalies in patients presenting with pectus deformities. J Pediatr Surg 2019;54:1926-8.

14. Rudbeck-Resdal J, Christiansen MK, Johansen JB, et al. Aetiologies and temporal trends of atrioventricular block in young patients: a 20-year nationwide study. Europace 2019;21:1710-6.

15. Kusumoto FM, Schoenfeld MH, Barrett C, et al. 2018 ACC/AHA/HRS Guideline on the Evaluation and Management of Patients With Bradycardia and Cardiac Conduction Delay: A Report of the American College of Cardiology/American Heart Association Task Force on Clinical Practice Guidelines and the Heart Rhythm Society. Circulation 2019;140:e382-482.

Cite this article as: Wang G, Yu Z, Zhang C, Zang H, Monti L, Jeong JY, Schmid RA, Pilegaard HK, Liu H. Pectus excavatum requiring temporary pacemaker implantation before Nuss procedure: a case report. J Thorac Dis 2020;12(9):4985-4990. doi: $10.21037 /$ jtd-20-2312 